\title{
Acoustic Birefringence and Poisson's Ratio Determined by Ultrasound: Tools to Follow-Up Deformation by Cold Rolling and Recrystallization
}

\author{
Linton Carvajal ${ }^{a *}$, Alfredo Artigas ${ }^{a}$, Alberto Monsalve ${ }^{a}$, Yolanda Vargas ${ }^{b}$ \\ ${ }^{a}$ Department of Metallurgical Engineering, Universidad de Santiago de Chile, Av. Libertador Bernardo \\ O'Higgins, 3363, Estación Central, Santiago, Chile. \\ ${ }^{b}$ Department of Physics, Universidad de Santiago de Chile, Av. Libertador Bernardo O'Higgins, 3363, \\ Estación Central, Santiago, Chile.
}

Received: December 13, 2016; Revised: June 25, 2017; Accepted: July 5, 2017

\begin{abstract}
Poisson's ratio and birefringence, both measured by ultrasound, are used to follow the evolution of the anisotropy in ASTM A-36 steel plates cold-rolled between 5 and $50 \%$ deformation, and then subjected to recrystallization at 900 and $1000{ }^{\circ} \mathrm{C}$. Times of flight of longitudinal and shear waves along the thickness of the plates were measured. As orthotropy increases, both birefringence and the difference between Poisson's ratios measured using a shear wave polarized along the length and another wave polarized along the width of the plate, are linearly related to the degree of deformation and cold-rolled hardness. In addition, the ultrasonic methods used clearly detected the complex changes in anisotropy produced by the austenization and recrystallization heat treatments. Thus, Birefringence or Poisson's ratio, measured by ultrasound, can be used to follow-up nondestructively changes in the anisotropy of rolled plates as a function of both, degree of deformation and recrystallization heat treatment.
\end{abstract}

Keywords: Ultrasound, Poisson's Ratio, Acoustic Birefringence, Cold Working, Recrystallization

\section{Introduction}

The use of ultrasound to measure second order elastic constants in multiphase or polycrystalline materials is based on the Christoffel equation, which relates them with the phase velocity of three non-dispersive ultrasonic waves with mutually perpendicular polarization directions (i.e., of displacement of the medium's particles), propagating in specified directions ${ }^{1}$. This equation comes from considering that the passage of a wave through a body generates small elastic stresses and deformations, setting up a dynamic equilibrium described by the equations of motion, wherefrom the equation that governs wave propagation in a homogeneous elastic medium is obtained by considering the generalized form of Hooke's law. The Christoffel equation is finally obtained by assuming the propagation of harmonic elastic waves as a solution for the equation of motion:

$$
\left(C_{i j k l} n_{j} n_{k}-\rho v^{2} \delta_{i l}\right) u_{l}=0
$$

where $\mathrm{C}_{\mathrm{ijk}}$ are the second order elastic constants; $\left(\mathrm{n}_{1}, \mathrm{n}_{2}, \mathrm{n}_{3}\right)$, the direction cosines of the normal to the wavefront, indicating, therefore, the direction of propagation of the wave; $u_{1}$ is the displacement or polarization vector; $\rho$, the density of the medium, and $\delta_{\mathrm{il}}$, the Kroenecker delta. The equation corresponds to three homogeneous equations from which, for every propagation direction considered, three different velocity values arise from the cubic equation in $\mathrm{v}^{2}$, obtained by making the determinant of the coefficient matrix equal to zero. These three velocities correspond to three waves with mutually perpendicular polarization vectors.

The solution to the inverse problem of determining the elastic constants from experimental measurements of wave velocity is well established, and the measuring protocols are clearly defined in the literature ${ }^{1}$. Most materials used in engineering either have isotropic symmetry, with two independent elastic constants, or orthotropic symmetry, with nine independent constants. The following relations are obtained for the isotropic case, where $\mathrm{C}_{11}=\mathrm{C}_{22}=\mathrm{C}_{33}$, $\mathrm{C}_{44}=\mathrm{C}_{55}=\mathrm{C}_{66}=1 / 2\left(\mathrm{C}_{11}-\mathrm{C}_{12}\right)$ :

$$
\begin{aligned}
& \mathrm{C}_{11}=\rho \mathrm{v}_{i i}^{2} \equiv \rho \mathrm{v}_{\mathrm{L}}^{2} ; \quad \mathrm{C}_{44}=\rho \mathrm{v}_{i j}^{2} \equiv \\
& \rho \mathrm{v}_{\mathrm{T}}^{2} ; \quad \mathrm{C}_{12}=\rho\left(\mathrm{v}_{\mathrm{L}}^{2}-2 \mathrm{v}_{\mathrm{T}}^{2}\right) ;
\end{aligned}
$$

with $\mathrm{v}_{i i}$, the velocity of the longitudinal wave (longitudinally polarized to its direction of propagation $i$ ), and $\mathrm{v}_{i j}$, with $i \neq j$, the velocity of the shear wave (polarized in the direction $j$, transverse to its direction of propagation $i$ ). Thus, the values of the elastic constants may be obtained just by measuring the velocities of a longitudinal wave and a shear wave in any direction of propagation. From the theory of elasticity ${ }^{2}$, which for the isotropic case relates the elastic stiffness constants with Young's and shear moduli, and with Poisson's ratio $v$, these parameters may be expressed in terms of the velocities 
of the longitudinal and shear waves ${ }^{3}$, as Equation (3) shows for Poisson's ratio:

$$
\nu=\frac{\left(\mathrm{v}_{\mathrm{L}} / \mathrm{v}_{\mathrm{T}}\right)^{2}-2}{2\left[\left(\mathrm{v}_{\mathrm{L}} / \mathrm{v}_{\mathrm{T}}\right)^{2}-1\right]}
$$

Since in an isotropic material $\mathrm{v}_{\mathrm{L}}$ and $\mathrm{v}_{\mathrm{T}}$ are independent of their directions of propagation and polarization, access to any one plane is enough to calculate its elastic properties.

In order to obtain the independent constants of an orthotropic material, nine measurements are required. These are the velocities of longitudinal and shear waves in the three symmetry directions of the material, allowing to obtain $\mathrm{C}_{11}, \mathrm{C}_{22}, \mathrm{C}_{33}, \mathrm{C}_{44}, \mathrm{C}_{55}$ and $\mathrm{C}_{66}$; and the velocities of either quasi-longitudinal or quasi-shear waves in the three symmetry planes, but at an angle with respect to the axes, obtaining $\mathrm{C}_{12}, \mathrm{C}_{13}$ and $\mathrm{C}_{23}$. Now, using only normal incidence for propagation along the z-axis (Figure 1), the following relations are obtained:

$$
\mathrm{C}_{33}=\rho \mathrm{v}_{33}^{2} ; \mathrm{C}_{44}=\rho \mathrm{v}_{32}^{2} ; \mathrm{C}_{55}=\rho \mathrm{v}_{31}^{2}
$$

That is, although to obtain all of the constants of an orthotropic material, three perpendicular planes must necessarily be accessed, variations in the degree of orthotropy may be studied by having access to only one plane and measuring the velocities of the shear waves $\mathrm{v}_{31}$ and $\mathrm{v}_{32}$, thus determining the difference between the elastic constants $\mathrm{C}_{44}$ and $\mathrm{C}_{55}$. This difference gives rise to the acoustic birefringence $\mathrm{B}$, which is quantified as the ratio of the difference of velocities $v_{31}$ and $\mathrm{v}_{32}$ to their average, as Equation 5 shows, so that for a perfectly isotropic material, $\mathrm{B}=0$ :

$$
B=\frac{v_{31}-v_{32}}{\frac{1}{2}\left(v_{31}+v_{32}\right)}=\frac{t_{32}-t_{31}}{\frac{1}{2}\left(t_{32}+t_{31}\right)}
$$

where $t_{i j}$ corresponds to the times of flight of the waves along the z-axis. The equation shows that birefringence can be quantified without measuring the distance, usually the thickness as in the case of rolled metal plates, of the material through which the wave travels, because the ratio of the velocities of two waves traveling the same distance becomes a ratio of the times of flight of those waves. Therefore, on the one hand, an important source of error in measurement is omitted, and on the other hand, it allows the study to be made under conditions in which it is difficult or impossible to measure the thickness ${ }^{4-7}$.

Notice that Equation (3) indicates that the thickness can also be omitted to calculate Poisson's ratio. This equation is valid for an isotropic material; for an orthotropic material, the difference between the elastic constants $\mathrm{C}_{44}$ and $\mathrm{C}_{55}$ should generate two values for Poisson's ratio, depending on the polarization direction of the shear wave, that is:

$$
\begin{aligned}
& \nu_{31}=\frac{\left(\mathrm{v}_{33} / \mathrm{v}_{31}\right)^{2}-2}{2\left[\left(\mathrm{v}_{33} / \mathrm{v}_{31}\right)^{2}-1\right]}=\frac{\left(\mathrm{t}_{31} / \mathrm{t}_{33}\right)^{2}-2}{2\left[\left(\mathrm{t}_{31} / \mathrm{t}_{33}\right)^{2}-1\right]} \\
& \nu_{32}=\frac{\left(\mathrm{v}_{33} / \mathrm{v}_{32}\right)^{2}-2}{2\left[\left(\mathrm{v}_{33} / \mathrm{v}_{32}\right)^{2}-1\right]}=\frac{\left(\mathrm{t}_{32} / \mathrm{t}_{33}\right)^{2}-2}{2\left[\left(\mathrm{t}_{32} / \mathrm{t}_{33}\right)^{2}-1\right]}
\end{aligned}
$$

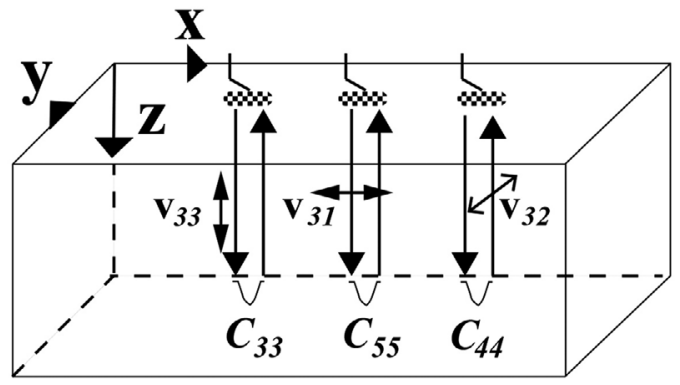

Figure 1. Waves for the determination of elastic constants, with longitudinal and shear waves propagating along $z$. The longitudinal wave is polarized along the $\mathrm{z}$-axis, while the shear waves of velocities $\mathrm{v}_{31}$ and $\mathrm{v}_{32}$ are polarized along the $\mathrm{x}$ and $\mathrm{y}$ axes, respectively.

Therefore, it should be possible to relate Poisson's ratio with the effect of deformation processes and post deformation heat treatments on the anisotropy, without specifying the thickness of the material under study. There is no information in the literature with respect to the use of Poisson's ratio for this purpose. The objective of this paper is, then, to study the feasibility of relating Poisson's ratio and acoustic birefringence measured by ultrasound with the degree of cold-working and the temperature and time of austenization after cold deformation of an structural steel. For this research, the traditional contact ultrasonic technique was used (as Figure 1 suggests), which readily lends itself to some industrial use, though it might be a limitation when contact between transducers and the part becomes an issue. However, the method is suitable for any non-contact technique, such as Electromagnetic Acoustic Transducer (EMAT) and Laser Ultrasonic (LUS), as long as longitudinal and shear waves may be induced in the part.

\section{Experimental Procedure}

Seven samples measuring $505 \mathrm{~mm}$ x $54 \mathrm{~mm}$ x $15 \mathrm{~mm}$ were cut for cold rolling from a hot-rolled ASTM A-36 steel plate. Their surfaces were adequately prepared to readily accommodate the transducers and propagate the ultrasonic waves. Before rolling each sample, 21 points were identified along the central line of the top surface, separated by 10 $\mathrm{mm}$ each. The thickness at every point was measured with a micrometer. Then, the seven samples were rolled each at a different degree of deformation. Afterwards, the seven rolled samples were each cut into four equal parts, all of which were heat-treated in a $20-\mathrm{kW}$ Lindberg model 56667-E furnace, 
previously heated at the required temperature as shown in Table 1. Thus, four heat treatments were performed for each degree of deformation.

Table 1. Time and temperature for heat treatment of rolled samples.

\begin{tabular}{lcccc}
\hline Temp. ${ }^{\circ} \mathrm{C}$ & 900 & 900 & 1000 & 1000 \\
\hline Time, min. & 15 & 30 & 15 & 30 \\
\hline
\end{tabular}

To determine birefringence and Poisson's ratio, normal incidence from longitudinal and shear contact piezoelectric transducers and pulse-echo technique were used. Ultrasonic tests were carried out before and after each deformation and after each heat treatment in the 21 locations corresponding to the marked points. The waves propagated from the top surface (xy plane in Figure 1) and along the thickness of the samples (z-axis in Figure 1). Naturally, longitudinal waves were always polarized along the $\mathrm{z}$-axis. On the other hand, by alternatively placing the shear wave transducer on the surface with its polarization direction perpendicular to and along the rolling direction, shear waves polarized along the principal axes ( $\mathrm{x}$ and $\mathrm{y}$ axes in Figure 1) of the samples were propagated. The ultrasonic system consisted of a Panametrics 5077PR pulser-receiver, on pulse-echo mode; 5-MHz, $11 \mathrm{~mm}$ in diameter Panametrics contact transducers for longitudinal and shear waves; and an HS805 TiePie oscilloscope emulator plate to obtain and store the echoes for later processing.

To relate results to standard techniques, metallographic samples were prepared by common procedures, and Rockwell $\mathrm{B}$ hardness was measured before and after deforming the samples, and after the heat treatments. Five measures were taken on each sample for averaging.

\section{Results and Discussion}

\subsection{Effect of cold rolling on wave velocities}

After obtaining and storing the ultrasonic signals, the times of flight of the waves were measured, quantified as the difference between the times of the maxima of the first two echoes.

Table 2 shows the propagation velocity values before and after rolling the samples; each value corresponds to the average of the measurements for each of the 21 points marked along the samples before deformation.
The longitudinal velocity $\mathrm{v}_{33}$, whose polarization vector is perpendicular to the rolling direction, has a decreasing trend as the degree of deformation increases beyond $17.9 \%$, reaching, at $42 \%$, a maximum difference of $46 \mathrm{~m} / \mathrm{s}$ $(0.78 \%)$ compared to its original average value without cold deformation. On the other hand, it is observed that for the original non-cold rolled sample, the difference between the average values of velocities of the perpendicularly polarized shear waves, $v_{31}$ and $v_{32}$, is barely $3 \mathrm{~m} / \mathrm{s}$. From Equation 4 , this gives rise to a difference of a mere $0.18 \%$ between $\mathrm{C}_{44}$ and $\mathrm{C}_{55}$, rendering the sample nearly isotropic. As cold deformation increases, the shear wave velocity $\mathrm{v}_{31}$, polarized parallel to the rolling direction, increases constantly from its original average value, so that the velocity is $2 \mathrm{~m} / \mathrm{s}$ higher $(0.06 \%)$, at $\varepsilon=5.4 \%$ and $28 \mathrm{~m} / \mathrm{s}$ higher $(0.86 \%)$ at $\varepsilon$ $=49.7 \%$; meanwhile, $\mathrm{v}_{32}$ (perpendicularly polarized to the rolling direction) decreases between 5 and $86 \mathrm{~m} / \mathrm{s}(0.15 \%$ and $2.65 \%$ ), relative to its respective original value. Following Equation 4, this implies that $\mathrm{C}_{55}$ increases and $\mathrm{C}_{44}$ decreases because of the deformation, while the effect on $\mathrm{C}_{33}$ is more complex, although it clearly tends to decrease.

\subsection{Effect of cold rolling on poisson's ratio and birefringence}

According to Equations 5, 6 and 7, the changes in the elastic constants modify B and $v$. To illustrate the effect of cold rolling, Figure 2a compares Poisson's ratios at the 21 locations of a sample before and after cold rolled to $12.3 \%$ deformation, and Figure $2 \mathrm{~b}$ does the same for the sample rolled to $42 \%$. On the non-rolled samples of Figure 2, the average values of Poisson's ratios determined from Equations 6 and 7 are $\bar{v}_{31}=0.2817$ and $\bar{v}_{32}=0.2810$, respectively. Figure 2 also shows that the individual values of $v_{31}$ and $v_{32}$ before deformation slightly fluctuate around their respective mean values, with a maximum difference between $v_{31}$ and $v_{32}$ of 0.0019 at locations 4 and 10. On the other hand, when the samples are rolled, fluctuations around the respective averages increase somehow, but more importantly, as the deformation increases, $v_{31}$ decreases while $v_{32}$ increases at every location. Hence, the difference between the mean values for the sample rolled to $12.3 \%$ grows to $\bar{v}_{31}-\bar{v}_{32}=0.0042$, while for the one rolled to $42 \%$, climbs to $\overline{v_{31}}-\overline{v_{32}}=0.0168$, that is, more than an order of magnitude greater than $\bar{v}_{31}-\bar{v}_{32}=-0.0007$, the difference between the mean values for the non-rolled samples. The effect of cold deformation, from 0 to $49.7 \%$,

Table 2. Averages of the wave velocities and hardness in the rolled samples.

\begin{tabular}{lcccccccc}
\hline Sample & Original & 1 & 2 & 3 & 4 & 5 & 6 & 7 \\
\hline Deformation $\varepsilon(\%)$ & 0.0 & 5.4 & 12.3 & 17.9 & 21.2 & 30.8 & 42.0 & 49.7 \\
$\mathrm{~V}_{33}(\mathrm{~m} / \mathrm{s})$ & 5882 & 5905 & 5884 & 5890 & 5866 & 5843 & 5836 & 5859 \\
$\mathrm{v}_{31}(\mathrm{~m} / \mathrm{s})$ & 3243 & 3245 & 3247 & 3251 & 3254 & 3250 & 3261 & 3271 \\
$\mathrm{v}_{32}(\mathrm{~m} / \mathrm{s})$ & 3246 & 3241 & 3225 & 3215 & 3206 & 3194 & 3174 & 3160 \\
$\mathrm{H}(\mathrm{HRB})$ & 82 & 90 & 92 & 93 & 94 & 95 & 98 & 101 \\
\hline
\end{tabular}



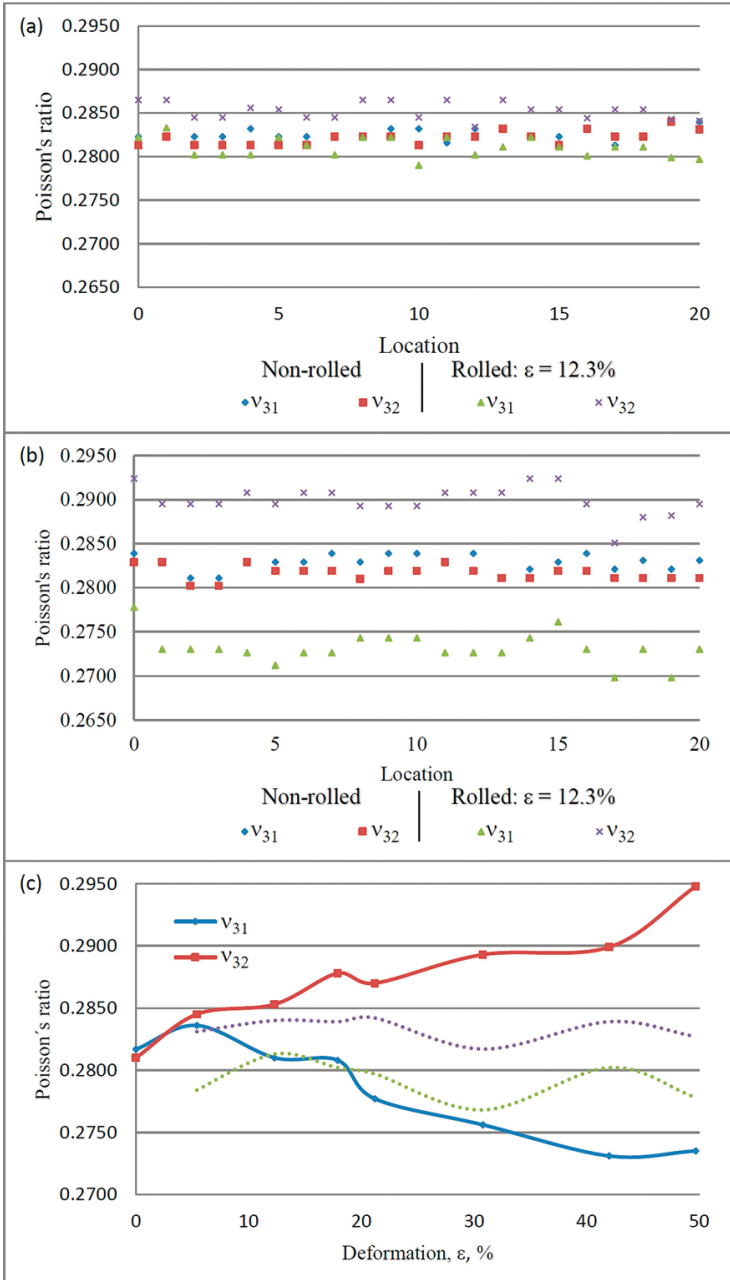

Figure 2. Poisson's ratios measured at 21 locations on samples; (a) non-cold rolled and rolled to $\varepsilon=12.3 \%$; (b) non-cold rolled and rolled to $\varepsilon=42 \%$. (c) Average of Poisson's ratios $v_{31}$ and $v_{32}$ versus cold deformation (dotted lines indicate the range of fluctuation of values of $v_{31}$ and $v_{32}$ before rolling).

on the average values of $v_{31}$ and $v_{32}$ is shown in Figure 2c. For comparison, the dotted lines also shown indicate the range of fluctuation of the individual values of $v_{31}$ and $v_{32}$ along the 21 points measured on each of the seven samples before rolling.

Figure 3 summarizes the effect of cold rolling on the difference between average values of Poisson's ratios (that is, the difference between the curves of Figure 2c), and on birefringence. It is seen that $\Delta v=\left(\bar{v}_{31}-\bar{v}_{32}\right)$ increases linearly with deformation from -0.0007 to 0.0213 . Likewise, the average birefringence $B$, which is barely -0.0011 in the hot rolled condition, reflecting its nearly isotropic behavior, increases linearly with cold deformation to 0.0346 . Equations (8) and (9) give the respective relations:

$$
\begin{aligned}
& \Delta \nu=\left(\bar{\nu}_{31}-\bar{\nu}_{32}\right)=0.0004 \varepsilon- \\
& 0.0009 \quad r^{2}=0.9932
\end{aligned}
$$

$$
B=0.0007 \varepsilon-0.0017 \quad r^{2}=0.9871
$$

Thus, it is possible to establish a linear relation between the difference of Poisson's ratios and birefringence. Such relation is given by:

$$
\begin{aligned}
& \Delta \nu=\left(\bar{\nu}_{31}-\bar{\nu}_{32}\right)=0.6215 B+ \\
& 0.0004 \quad r^{2}=0.9761
\end{aligned}
$$

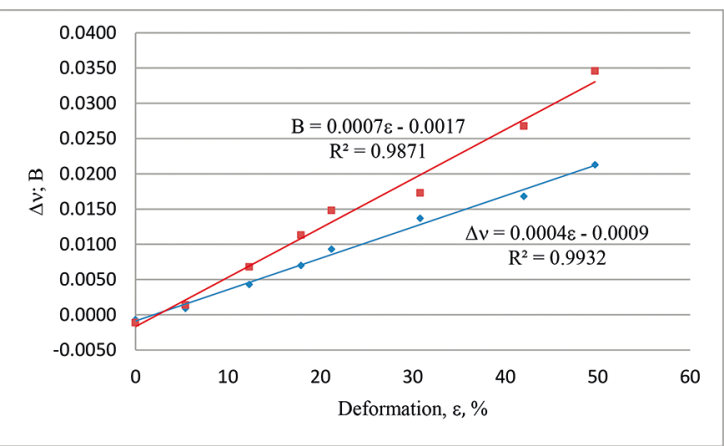

Figure 3. Effect of deformation on birefringence $B$ and on the difference between Poisson's ratios $\Delta v=\left(\overline{v_{31}}-\overline{v_{32}}\right)$.

\subsubsection{Relationship to hardness}

Average values of hardness for the samples are shown in Table 2. The average value for the original hot rolled plate was 82 (HRB). As expected, hardness increases with cold rolling, and from the data in Table 2 is seen that it does it linearly as deformation increases between 5.4 and $49.7 \%$. The resultant relation is given by Equation (11), where, $\mathrm{H}$ stands for hardness.

$$
H=0.2302 \varepsilon+88.8 \quad r^{2}=0.978
$$

As a consequence, both birefringence and the difference of Poisson's ratios are linearly related to hardness. The respective relations are given by:

$$
\begin{gathered}
B=0.0031 H-0.2739 \quad r^{2}=0.9961 \\
\Delta \nu=\left(\bar{\nu}_{31}-\bar{\nu}_{32}\right)=0.0019 H- \\
0.1658 \quad r^{2}=0.9649
\end{gathered}
$$

Therefore, it is possible to estimate precisely the level of deformation as well as the hardness achieved by rolling from measurements of the time of flight of shear waves for birefringence, and of longitudinal and shear waves for Poisson's ratios. 


\subsection{Effect of heat treatment on poisson's ratio and birefringence}

For high levels of deformation, Figures 4 and 5 show that the heat treatments to which the rolled samples were subjected tend to return the values of Poisson's ratio to the original values, which are shown by the dotted lines that indicate the range of fluctuation of the ratios, both $v_{31}$ and $v_{32}$, considering the 21 points measured on each of the seven samples before rolling. However, at $900^{\circ} \mathrm{C}$ (Figures $4 \mathrm{a}$ and $4 \mathrm{~b}$ ), it is seen that regardless of treatment time, between $17.9 \%$ and $30.8 \%$ deformations, the $v_{31}$ curves show a maximum, shifting even more from the original values. This implies that the material behaves more anisotropically after the heat treatment within that deformation range.
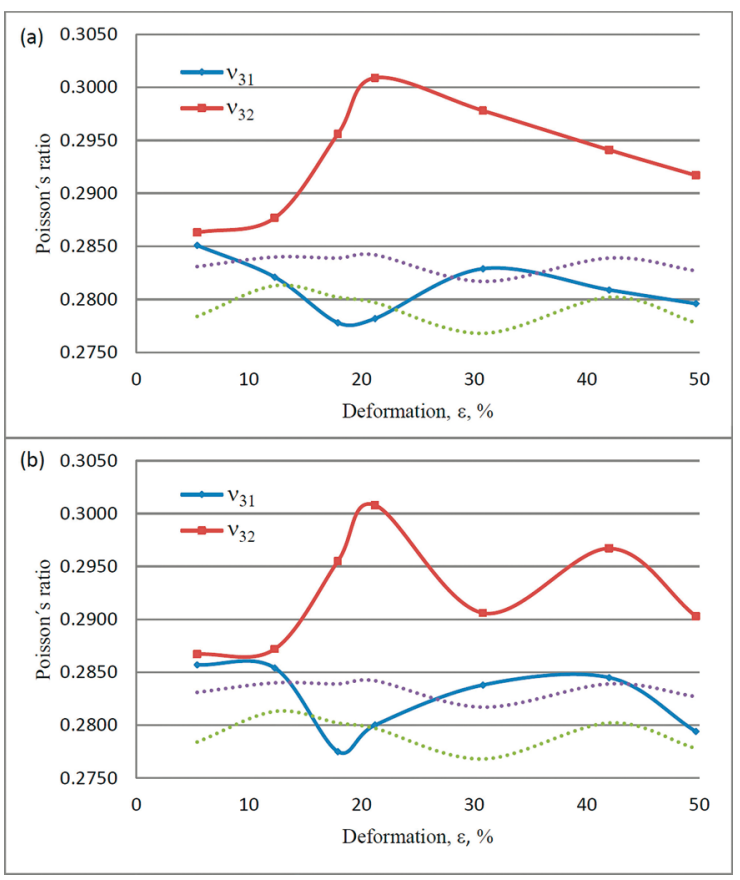

Figure 4. Effect of heat treatment on Poisson's ratio $v_{31}$ and $v_{32}$ in rolled samples. (a) $900^{\circ} \mathrm{C}, 15 \mathrm{~min}$.; (b) $900^{\circ} \mathrm{C}, 30 \mathrm{~min}$. (Dotted lines indicate the range of fluctuation of values of $v_{31}$ and $v_{32}$ before rolling).

The tests carried out at $1000{ }^{\circ} \mathrm{C}$ (Figures 5a and 5b) show a behavior similar to the previous one, but only for a time of 15 minutes of treatment (Figure 5a). It is also seen that for deformations lower than $17.9 \%$ and greater than $30.8 \%, v_{31}$ and $v_{32}$ are practically identical.

Therefore, only for the treatment at $1000{ }^{\circ} \mathrm{C}$ for 30 minutes (Figure 5b), the samples, regardless of the degree of deformation to which they were subjected, return to a practically isotropic behavior.

Those results may be explained as follows: it would be expected that a cold-deformed ASTM A-36 steel sample, when heated above the upper critical temperature curve,
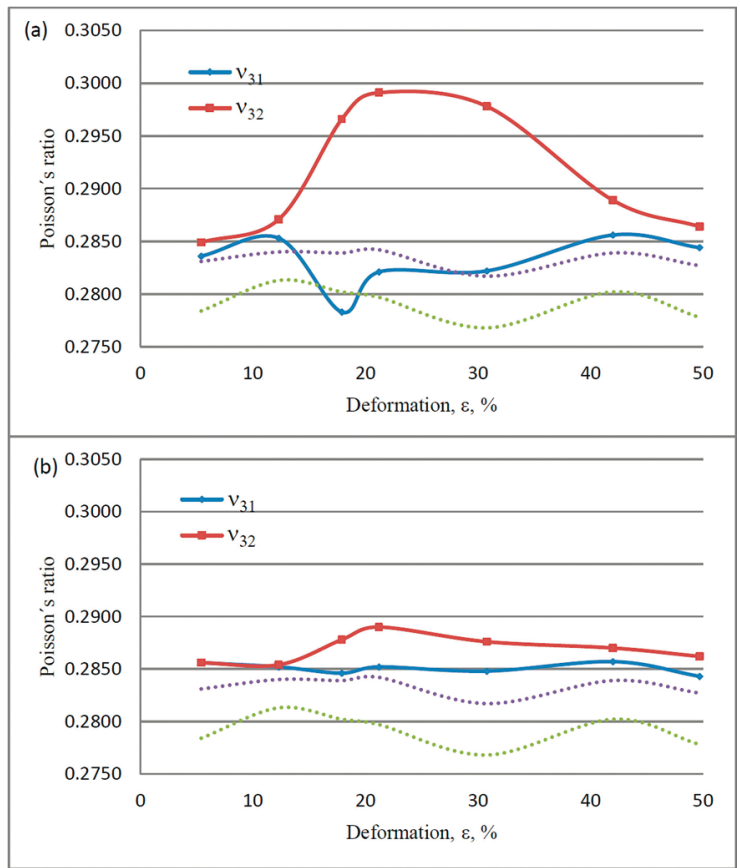

Figure 5. Effect of heat treatment on Poisson's ratio $v_{31}$ and $v_{32}$ in rolled samples. (a) $1000{ }^{\circ} \mathrm{C}, 15 \mathrm{~min}$.; (b) $1000^{\circ} \mathrm{C}, 30 \mathrm{~min}$. (Dotted lines indicate the range of fluctuation of the individual values of $v_{31}$ and $v_{32}$ among the 21 points measured on each sample before rolling).

would recrystallize below curve $A_{1}$, followed by a partial transformation above curve $\mathrm{A}_{1}$ and a total transformation above curve $\mathrm{A}_{3}$. Then, on cooling the $\gamma \rightarrow \alpha$ and $\gamma \rightarrow \alpha$ + cementite transformations would occur. The onset of recrystallization and the recrystallized fraction depend on both the degree of deformation applied and the time at the recrystallization temperature. For low levels of deformation, the $\alpha \rightarrow \gamma \rightarrow \alpha$ transformations could take place without prior recrystallization. If so is the case, the deformed $\alpha$ structure $\left(\alpha_{D E F}\right)$ can be expected to be transformed into deformed $\gamma$ $\left(\gamma_{D E F}\right)$ upon heat treatment, and then on cooling it would transform back into $\alpha_{D E F}$.

Then, graphs a and b of Figure 4 suggest that up to $21.2 \%$ deformation, at $900{ }^{\circ} \mathrm{C}$ the $\alpha_{D E F} \rightarrow \gamma_{D E F} \rightarrow \alpha_{D E F}$ transformation cycle may take place, whether the heat treatment lasts 15 or 30 minutes.

At $1000^{\circ} \mathrm{C}$, on the other hand, the $\alpha_{D E F} \rightarrow \gamma_{D E F} \rightarrow \alpha_{D E F}$ transformation cycle is valid only for 15 minutes of heat treatment. Apparently, as time at $1000{ }^{\circ} \mathrm{C}$ is increased all the $\gamma$ grains would adopt a gray texture, giving rise to $\alpha$ grains without a preferred orientation. The same would happen with $30.8 \%$ deformation at the two heat treatment temperatures. The analysis suggests that for $42 \%$ deformation at $900{ }^{\circ} \mathrm{C}$ (Figures $4 \mathrm{a}$ and $4 \mathrm{~b}$ ) the $\alpha_{D E F} \rightarrow \gamma_{D E F} \rightarrow \alpha_{D E F}$ cycle still takes place in a time of 15 minutes, however, at a temperature of $1000^{\circ} \mathrm{C}$ (Figures 5a and 5b) there would be recrystallization prior to the $\alpha \rightarrow \gamma$ transformation. A $49.70 \%$ deformation is 
apparently sufficient to produce some degree of recrystallization prior to the $\alpha \rightarrow \gamma$ transformation, because it is seen that the values tend toward those of the isotropic material. This practically $100 \%$ isotropic symmetry is achieved after 30 minutes at $1000{ }^{\circ} \mathrm{C}$ for all degrees of deformation, as shown in Figure 5b, where Poisson's ratios tend to 0.286 , a value slightly higher than the range of the original samples from the hot rolled material.

As could be expected, the behavior of birefringence to the heat treatments is similar to that of Poisson's ratio.

\subsection{Hardness and metallographic analysis}

The micrographs shown in this section were taken at a magnification of 500X. Figure $6 \mathrm{a}$ belongs to a material in its original state, showing an equiaxial ferrite plus pearlite microstructure, typical of an ASTM A-36 steel. Figure 6b shows a micrograph of a rolled sample at a medium degree of deformation, where, as expected, no significant change is seen in the orientation of the grains. As is well known, more sophisticated techniques, such as DRX and EBDS, are required to detect such changes. However, the results obtained by ultrasound clearly show that it is possible, in a nondestructive mode, to detect the increased orthotropy at even the lowest degrees of deformation.

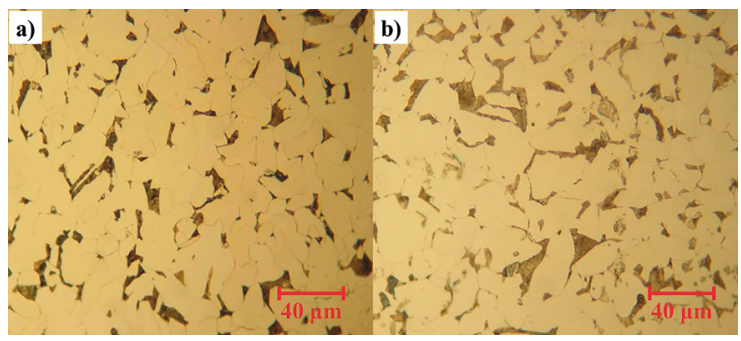

Figure 6. a) Material in its original state; b) sample rolled to $21.2 \%$.

Figures $7 \mathrm{a}$ and $7 \mathrm{~b}$ show that after the heat treatments there is a slight difference in ferritic grain size compared to the original sample (Figure 6a), and that in the sample treated at higher temperature there is the presence of colonies of thicker pearlite.

The hardness of all the heat-treated samples fluctuated randomly between 77 and 82 (HRB), with no relation to the temperature or time of treatment, showing a decrease of the hardness down to values lower in most cases than the average value obtained for the original plate.

The previous hardness results and the metallographic analysis show that those procedures do not allow following up the process of modification of anisotropy caused by the heat treatment applied to the cold-rolled samples. In contrast, the determination of birefringence or Poisson's ratio by ultrasonic methods allows these modifications to be distinguished precisely.

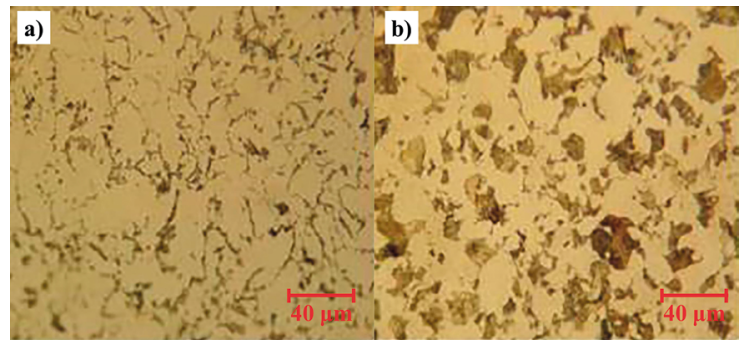

Figure 7. a) Sample rolled to $21.2 \%$, and annealed at $900{ }^{\circ} \mathrm{C}, 30$ min.; b) sample rolled to $49.7 \%$, and annealed at $1000{ }^{\circ} \mathrm{C}, 30 \mathrm{~min}$.

\section{Conclusions}

The main conclusions obtained from this work are the following:

- The mapping of Poisson's ratio on the ASTMA-36 steel showed it to be nearly isotropic.

- It was shown experimentally that the effect of deformation by cold rolling on wave velocity, birefringence, and Poisson's ratio is to shift the obtained data from their initial values in proportion to the degree of deformation applied.

- The above means that both birefringence and Poisson's ratio make it possible to determine the degree of cold deformation and the hardness of the materials studied by means of simple linear relationships.

- The effect of the heat treatment in the austenitic field on cold-rolled samples depends on the degree of deformation and on the temperature and time of treatment. Medium levels of deformation, low temperature and shorter austenization time tend to shift the values of birefringence and Poisson's ratio from the original values of the material before the deformation; high levels of deformation, high temperatures, and longer austenization tend to return those values to the original ones of the material before its deformation.

It can be concluded, therefore, that even though the times of flight of the waves measured in different positions show a random dispersion, it is feasible to use Poisson's ratio as well as birefringence, both measured by ultrasound, as a simple and nondestructive method to follow up the anisotropy changes in rolled plates due to cold rolling and to post rolling heat treatments.

\section{Acknowledgements}

The authors acknowledge with thanks the financial support provided by the Dirección de Investigaciones Científicas y Tecnológicas (DICYT) of the Universidad de Santiago de Chile and Proyecto Basal USA 1555 USACH-MECESUP for carrying out this work. 


\section{References}

1. Rose JL. Ultrasonic Waves in Solid Media. Cambridge: Cambridge University Press; 1999. p. 27-33, 272-285.

2. Dieter GE. Mechanical Metallurgy. London: McGraw-Hill; 1988. p. 26-60.

3. Bray DE, Stanley RK. Elastic Wave Propagation. In: Bray DE, Stanley RK. Nondestructive Evaluation. A Tool in Design, Manufacturing, and Service. Boca Raton: CRC Press; 1996. p. 53-59.

4. Schneider E. Ultrasonic Techniques. In: Hauk V. Structural and Residual Stress Analysis by Nondestructive Methods: Evaluation, Application, Assessment. Amsterdam: Elsevier; 1997. p. 523-563.
5. Spies M, Schneider E. Nondestructive Analysis of Textures in Rolled Sheets by Ultrasonic Techniques. Textures and Microstructures. 1990;12(4):219-231.

6. Sayers CM. Ultrasonic Determination of Texture and Residual Stress in Polycrystalline Metals. In: Alippi A, Mayer WG, eds. Ultrasonic Methods in Evaluation of Inhomogeneous Materials. Nato Science Series E: Applied Sciences, No 126. Dordrecht: Springer; 1987. p. 367-379.

7. Delsanto PP, Mignogna RB, Clark AV Jr. Ultrasonic texture and stress measurements in anisotropic polycrystalline aggregates. The Journal of the Acoustical Society of America. 1990;87(1):215-224. 